\title{
Resting membrane potential as a marker of apoptosis: studies on Xenopus oocytes microinjected with cytochrome $c$
}

\author{
AK Bhuyan ${ }^{1,2,3}$, A Varshney ${ }^{3}$ and MK Mathew ${ }^{*, 3}$ \\ ${ }^{1}$ Centre for Biochemical Technology (CSIR), Delhi University Campus, Delhi \\ 110007 , India \\ 2 Jawaharlal Nehru Centre for Advanced Scientific Research, Jakkur, Bangalore \\ 560065 , India \\ ${ }^{3}$ National Centre for Biological Sciences, TIFR Centre, UAS-GKVK Campus, \\ Bangalore 560 065, India \\ * Corresponding author: MK Mathew, National Centre for Biological Sciences, \\ TIFR Centre, UAS-GKVK Campus, Bangalore 560 065, India \\ Fax: 91-80-8561662; E-mail: mathew@ncbs.res.in
}

Received 14.1.00; revised 30.8.00; accepted 1.9.00

Edited by Y Tsujimoto

\begin{abstract}
Observation of the electrical potential difference across the cell membrane is described as a new method for monitoring apoptosis of a single cell. The resting membrane potential $(\Delta \Psi)$ of Xenopus oocytes has been recorded in real time following microinjection of cytochrome $c$. Soon after microinjection, $\Delta \Psi$ becomes less negative and attains a new constant value with a half time, $t_{m}$, of about $35( \pm 5)$ min at all cytochrome $c$ concentrations greater than $1 \mu \mathrm{M}$. The cytosol extract of cytochrome $c$-injected oocytes shows DEVD proteolytic activity characteristic of aspartate specific proteases, implicating an apoptotic death pathway. In response to the delivery of cytochrome $c$ into the cytosol, caspases are activated within 7 min while the changes in $\Delta \Psi$ begin to occur after about $30 \mathrm{~min}$. The method described here will be potentially useful to assess the effectiveness of cell death regulators and modulators of synthetic and biological origin, and the results presented shed light on the currently debated issue of the importance of the redox state of cytochrome $c$ in the initiation of apoptosis. Cell Death and Differentiation (2001) 8, 63-69.
\end{abstract}

Keywords: cytochrome $c$; apoptosis; Xenopus oocyte; membrane potential

Abbreviations: $\Delta \Psi$, resting membrane potential; Z-DEVD-AFC, Cbz-Asp-Glu-Val-Asp-amino-4-trifluoromethyl coumarin; AcDEVD-CHO, N-acetyl-Asp-Glu-Val-Asp-CHO (aldehyde); PBS, phosphate buffer saline; DMSO, dimethyl sulphoxide

\section{Introduction}

In metazoan cells cytochrome $c$ is known to activate the apoptotic program independent of the involvement of death ligands. $^{1-3}$ The death-promoting role of cytochrome $c$ has been demonstrated directly in a Xenopus cell-free apoptosis system where mitochondria spontaneously release cytochrome $c$ to activate caspases, leading to apoptotic nuclear morphology. ${ }^{4}$ Using a Xenopus cell-free system it has been shown that caspase 8 can act on mitochondria for translocation of cytochrome $c .^{5}$ The apoptotic program can also be set up artificially by delivering a load of exogenous cytochrome $c$ into the cytosol. Microinjection techniques have been used to add cytochrome $c$ into the cytosol of a variety of mammalian cells, including human embryonic kidney 293 cells, ${ }^{6}$ embryonic fibroblasts and tumor cells, ${ }^{7}$ and intact neurons, ${ }^{8}$ to show that the presence of the free form of cytochrome $c$ in the cytosol can initiate the apoptotic program.

Microinjection experiments can be used to explore various aspects of apoptosis, including morphological, biochemical, and physiological changes at different stages of cell death. The injected cells have hitherto been scored for apoptosis by microscopic observation, ${ }^{7}$ costaining and photography under ultraviolet illumination, ${ }^{6}$ and by antibody staining and immunofluorescence methods. ${ }^{8}$ In this communication we report on the use of the resting plasma membrane potential as a simple and reliable marker to follow apoptosis in a single cell with virtually no time delay after the delivery of cytochrome $c$. Initiation and propagation of apoptosis in intact Xenopus oocytes microinjected with cytochrome $c$ have been studied by recording the changes in resting membrane potential by using an intracellular microelectrode. Caspase involvement has been confirmed by DEVD proteolysis assay of cell-free extracts.

In this study we first establish that the plasma membrane depolarization in response to the cytosolic load of cytochrome $c$ is indeed a reliable marker of apoptotic death, and then study how long it takes for exogenously supplied cytochrome $c$ to activate caspases. Since the resolution of the temporal relationship of events observed during the apoptotic process could constrain models proposed for the operative pathway, we sought to determine whether the changes in the cell morphology and the cell membrane potential monitored in our experiments lead or lag caspase activation. The results suggest that caspases are activated within $7 \mathrm{~min}$ of cytosolic presence of cytochrome $c$, and both changes in electrical properties of the cell membrane and the cell surface morphology lag caspase activation. 


\section{Results and Discussion}

\section{Morphological features of oocytes following injection of cytochrome $c$}

Although cytochrome $c$-induced activation of caspases has been demonstrated in a Xenopus cell-free system, ${ }^{4,5}$ apoptosis of the intact oocyte has not been described. Therefore, we initially checked death in oocytes by direct microinjection of cytochrome $c$. Figure $1 \mathrm{~A}$ shows the deathpromoting effect of cytochrome $c$, manifested in the appearance of transparent blisters, the growth of irregular mottled patterns on the cell surface, and the gradual loss of the sharp distinction between the animal (dark or pigmented) and the vegetal (white) pole. These morphologic appearances, imaged $\sim 4.5 \mathrm{~h}$ after microinjection of cytochrome $c$, and which are due to the condensation of cytoplasmic material, ${ }^{3}$ were not observed when the microinjection protocol was simulated in control experiments (Figure 1B) where oocytes were injected with an equivalent volume of distilled water or with aqueous solutions of hemoglobin or barstar (a ribonuclease inhibitor protein the size of cytochrome $c$ ), suggesting that the observed features are the result of the load of cytochrome $c$ in the cytosol. Also, the surface morphology remains unaltered when cytochrome $c$ and AcDEVD-CHO, a caspase inhibitor, are coinjected (data not shown), implicating proteolytic activities of caspases for the observed changes in morphology.

\section{The kinetics of morphological changes following cytochrome $c$ injection}

The results of cellular microinjection experiments are generally reported as the number of apoptotic cells as a function of time. ${ }^{7}$ Clearly, the determination of the time dependence of the number of apoptotic cells based on qualitative observation of cellular morphology or of uptake of stain in a given set of induced cells, yields kinetics of population death. Some cells die early whereas others take a longer time. Figure 2 depicts the time course of the appearance of the number of mottled oocytes counting from the time of injection within a set of 24-synchronized microinjected cells. A useful parameter in this mode of presentation of results is the time for the half-maximal effect, $t_{1 / 2}$. It is noteworth that $t_{1 / 2}$ recorded in the present experiments, $2-3 \mathrm{~h}$ (Figure 2 ), is consistent with $t_{1 / 2}$ values read from the results of previous microinjection experiments on mammalian cell lines. ${ }^{6,7}$

\section{Real-time recording of cell membrane depolarization during cell death}

Since the physical integrity and the ion selectivity of the cell membrane maintain the electrical properties of the membrane, it occurred to us that the growth of the surface morphology, and hence the progress of cell death, could also be monitored in real time by recording the resting membrane potential of oocytes. We have used a glass micro-pipette to inject cytochrome $c$ into Xenopus oocytes, and subsequently recorded the potential across the cell membrane with an intracellular glass microelectrode. The time delay between
A

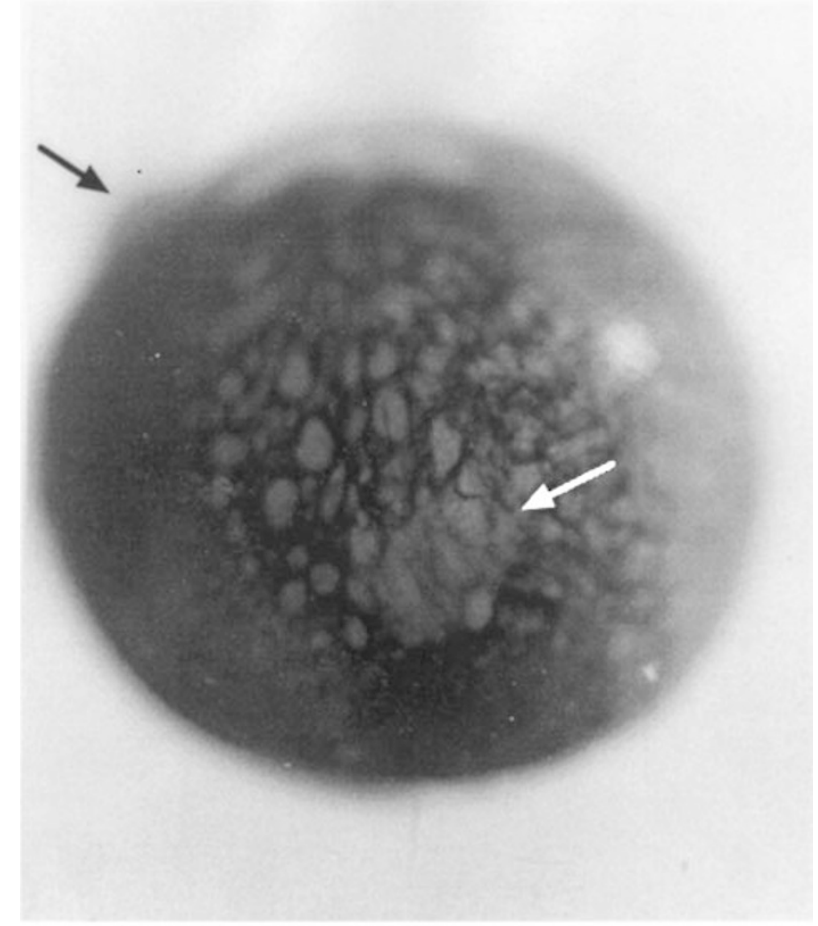

B

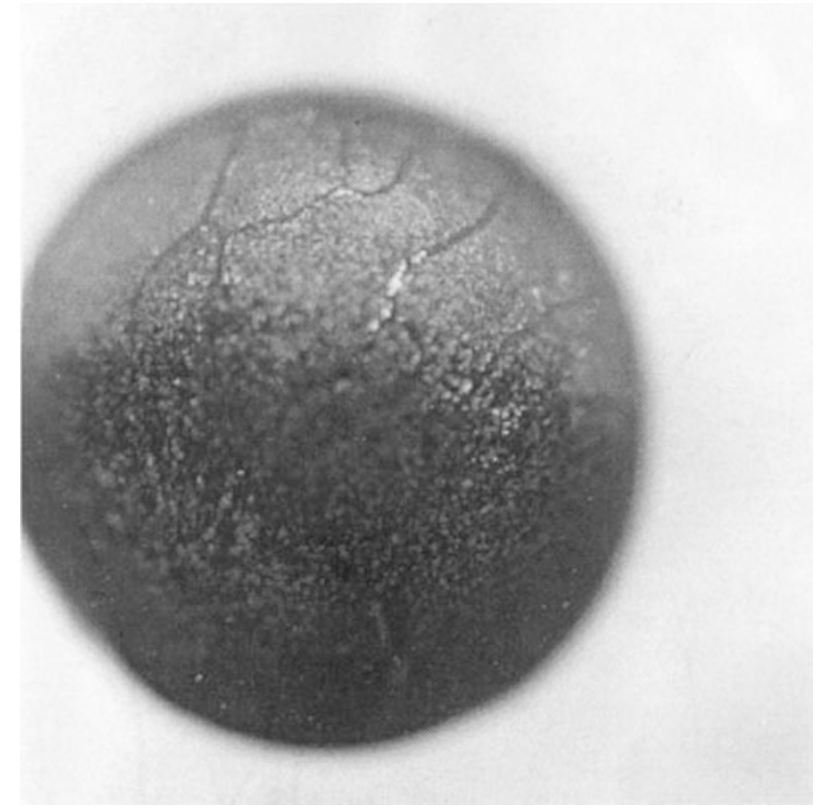

Figure 1 Death of Xenopus oocytes induced by the microinjection of cytochrome $c$. Both photographs were taken about $4.5 \mathrm{~h}$ after injection. (A) The general morphologic features associated with apoptosis appear in oocytes injected with cytochrome $c$ to a final concentration of $10 \mu \mathrm{M}$. The black arrow at the top left points to one of the more prominent blisters, while the white arrow in the middle of the oocytes highlights mottling of the surface of the animal pole. (B) Oocyte injected with an equal volume of a solution of a bacterial ribonuclease inhibitor (barstar). Oocytes injected with water, hemoglobin, and yeast iso-1-cytochrome $c$ also showed no significant morphological changes. Oocytes coinjected with $1.5 \mu \mathrm{M}$ cytochrome $c$ and $90 \mathrm{nM}$ Z-DEVD-CHO were similarly unaffected 


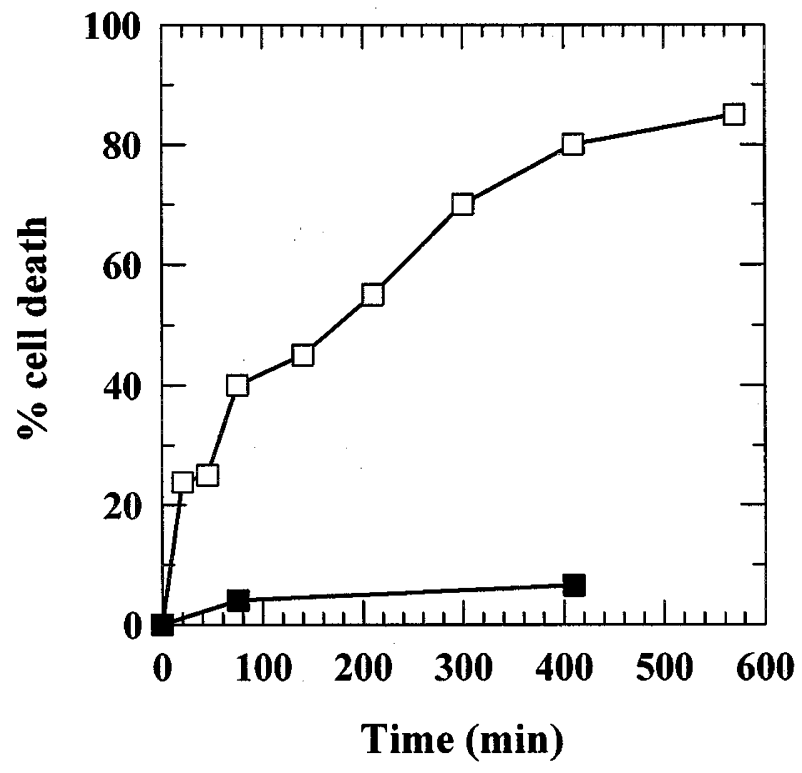

Figure 2 Visual record of the appearance of mottled patterns in a set of 24 oocytes microinjected with cytochrome $c(10 \mu \mathrm{M})$ synchronously ( $\square$ ), expressed as '\% cell death' as a function of time. In the control experiment, cytochrome $c$ was coinjected with $\sim 100 \mathrm{nM}$ (intracellular concentration) ZDEVD-CHO (a)

injection of cytochrome $c$ and electrode insertion, which we define as the dead time in the real time measurement of membrane potential, varies from 1 to $2 \mathrm{~min}$.

\section{Membrane potential in Xenopus oocytes during cell death}

We have recorded the potential across the plasma membrane of oocytes microinjected with cytochrome $c$ as a function of time following injection. Figure 3A shows real-time plots of $\Delta \Psi$ normalized with respect to the value recorded before the injection of cytochrome $c$. The initial dips in the profiles indicate recovery and restoration of the $\Delta \Psi$ to the original value after the injection shock. About 40 min after injection, the $\Delta \Psi$ begins to change to less negative values displaying a steep initial rise in the profile for about $40 \mathrm{~min}$ to reset to a new value $\sim 50 \%$ of the original. This new steady state is maintained for at least 2-3 h. $\Delta \Psi$ decays only slowly thereafter. At the end of $3 \mathrm{~h}, \Delta \Psi$ is close to $\sim 45 \%$ of the original value. The early decrease in $\Delta \Psi$, seen in several independent recordings using different batches of oocytes, is a discrete membrane depolarization event associated with cell death. In control runs, where oocytes were injected with an equal volume of distilled water or an aqueous solution of barstar or yeast iso-1-cytochrome $c, \Delta \Psi$ did not decrease to any appreciable extent (Figure $3 \mathrm{~A}$ ), suggesting that it is the load of cytochrome $c$ in the cytosol which is responsible for the observed change in membrane potential. The control experiment done with yeast iso-1-cytochrome $c$ particularly emphasizes the usefulness of the voltage probe method, since this isoform of cytochrome $c$ has been known to be nonapoptogenic (see, for example, reference ${ }^{9}$ ). To show that the
A

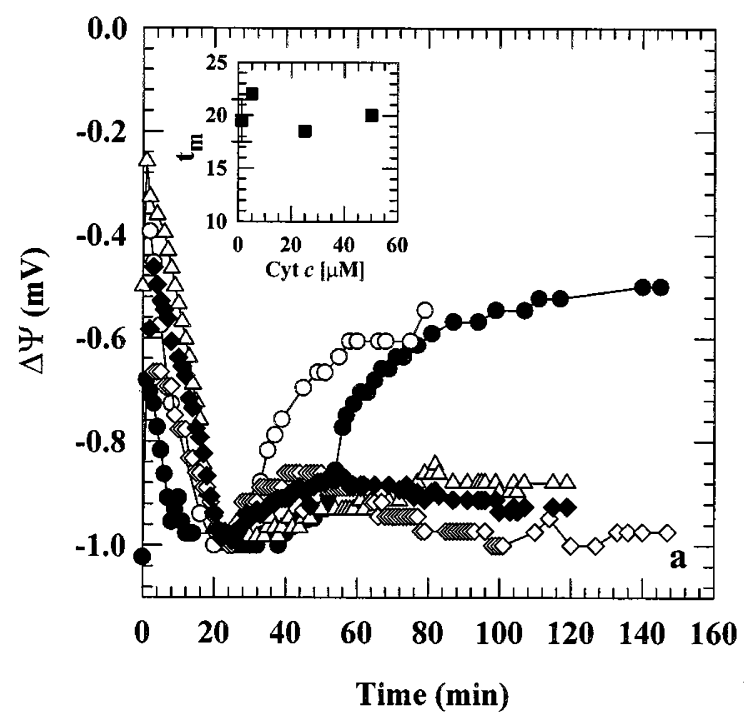

B

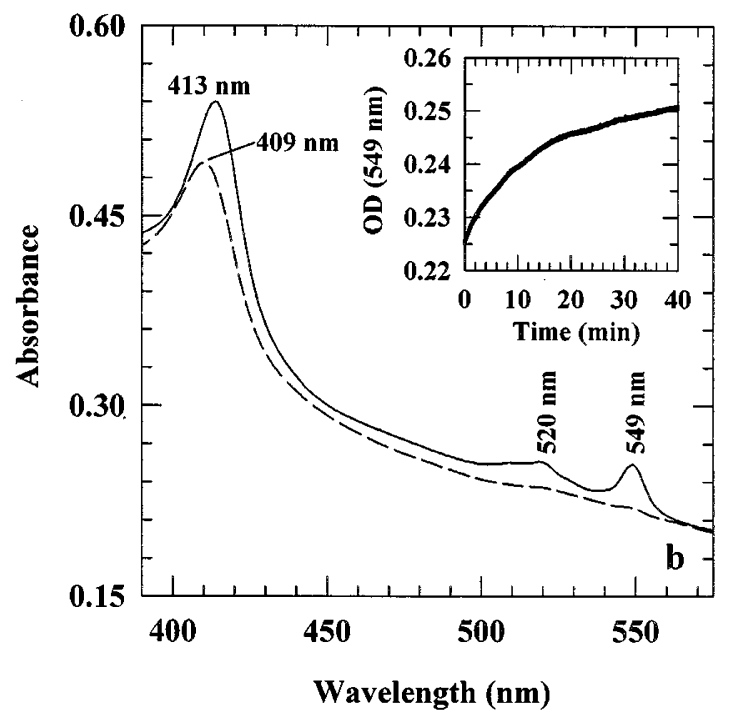

Figure 3 (A) Real-time recording of resting membrane potential $(\Delta \Psi)$ of oocytes injected with $4 \mu \mathrm{M}$ oxidized cytochrome $c(-), 4 \mu \mathrm{M}$ reduced cytochrome $c(\bigcirc), 25 \mu \mathrm{M}$ yeast iso-1-cytochrome $c(\diamond), 1.5 \mu \mathrm{M}$ cytochrome $c$ along with $90 \mathrm{nM} \mathrm{Ac-DEVD-CHO}(\diamond)$ and a $\sim 5 \mu \mathrm{M}$ ribonuclease inhibitor, barstar $(\Delta)$. The values of potential plotted on the $y$-axis have been arbitrarily normalized with respect to the value recorded for the oocyte before injection. The final intracellular concentrations of the injected proteins were closely comparable. Inset: cytochrome $c$ concentration independence of the time midpoint for the change of $\Delta \Psi$. The error bar shown for the lowest concentration of cytochrome $c$ has been estimated from two independent sets of observations. (B) Reduction of ferricytochrome $c$ by the oocyte cell-free extract. The initial spectrum of ferricytochrome $c$ (broken line) was recorded with the cell-free extract prepared immediately ( $\sim 2 \mathrm{~min})$ after microinjection of cytochrome $c$. The spectrum characteristic of ferrocytochrome $c$ (solid line) was recorded from an extract made from oocytes $\sim 60$ min after the injection of cytochrome $c$. Inset, kinetics of the appearance of ferrocytochrome $c$ monitored after addition of cytochrome $c(1.5 \mu \mathrm{M})$ to the extract of healthy oocytes $\left(K_{\mathrm{app}}=0.08 \mathrm{~min}^{-1}\right)$

observed change in membrane potential is due to apoptosis and activation of DEVDase induced by microinjection of cytochrome $c$, we recorded the time-dependent changes in 
$\Delta \Psi$ of oocytes after coinjection of Ac-DEVD-CHO, an inhibitor of caspase-3, and cytochrome $c$, such that their intracellular concentrations are $90 \mathrm{nM}$ and $1.5 \mu \mathrm{M}$, respectively (Figure $3 \mathrm{~A})$. The result was simple: the $\Delta \Psi$ was fully restored after injection shock recovery and there was no decay to less negative values.

The depolarization of the cell membrane potential indicates increased permeability of the membrane in cells following cytochrome $c$ injection. Although the available data do not permit a description at the molecular level for the development of membrane porosity and caspase activity, it is reasonable to assume that the condensation of the cytosolic material is related to the change in $\Delta \Psi$.

It needs to be mentioned that it is not only apoptotic death that is associated with a change in $\Delta \Psi$; death due to cell injury or necrosis also leads to membrane depolarization. For example, the membrane potential recorded after poisoning the electron transport chain by injecting $\mathrm{KCN}$ alone to a final intracellular concentration of $\sim 2 \mu \mathrm{M}$ shows a decay of $\Delta \Psi$ in a manner very similar to that observed with cytochrome $c$ injection (data not shown). The cytosolic extracts of these cells, however, fail to show DEVDase activity.

How is the cytosolic concentration of injected cytochrome $c$ related to the time needed for development of membrane depolarization? To answer this question we titrated the oocytes by microinjection of different concentrations of cytochrome $c$, and recorded their membrane potentials in a time-resolved manner. The intracellular concentration of injected cytochrome $c$ does not affect the time required for the observed shift in $\Delta \Psi$. This is shown in the plot of $t_{\mathrm{m}}$ vs intracellular concentration of cytochrome $c$ (in the range $1.5-50 \mu \mathrm{M}$ ), where $t_{\mathrm{m}}$ is the mid-point of the time taken for the change in $\Delta \Psi$ counted from the start of depolarization (inset, Figure $3 \mathrm{~A}$ ). When the intracellular concentration of cytochrome $c$ was lowered below $1 \mu \mathrm{M}$, the cell membrane remained polarized, suggesting insufficiency of such a low concentration for induction of apoptosis. This threshold value of cytochrome $c$ concentration appears intriguing, since only $\approx 0.05 \mu \mathrm{M}$ of cytochrome $c$ is needed to activate caspases in the Xenopus egg cell-free system. ${ }^{4}$ We do not know for sure why the observed threshold value of cytochrome $c$ for the intact Xenopus oocyte is so high. Since we have carried out in vivo experiments, it is possible that certain as yet unknown binder(s) of cytochrome $c$ reduce its effective cytosolic concentration available for caspase activation.

\section{Redox conformational requirement of cytochrome $c$ in the induction of apoptosis}

A strict requirement of the native conformation of cytochrome $c$ for the induction of apoptosis is now well established. Yang et al. ${ }^{10}$ showed the failure of apocytochrome $c$ in the induction of the apoptotic program, suggesting the obligatory requirement of the native conformation of holocytochrome $c$. A similar result that biotinylated cytochrome $c$ fails to induce apoptosis in normal rat kidney cells has also been reported. ${ }^{7}$ In the course of these experiments we also attempted to examine the effect of the two redox conformations of cytochrome $c$, ferricytochrome $c$ and ferrocytochrome $c$, on the time course of plasma membrane depolarization. The electron transfer function of cytochrome $c$ involves reversible transformation of the protein between the ferric $\left(\mathrm{Fe}^{3+}\right.$ and the ferrous $\left(\mathrm{Fe}^{2+}\right)$ form (ferricytochrome $c \leftrightarrow$ ferrocytochrome $c$ ). On the premise that the crystal structures of the two oxidation states of the holoprotein are very similar, ${ }^{11-13}$ it is expected that the role of cytochrome $c$ in apoptotic death would be independent of the redox state. An in vitro model indeed indicated that either oxidation state is effective in the activation of caspases. ${ }^{14}$ Two recent reports, however, appear to indicate that it is ferricytochrome $c$, and not ferrocytochrome $c$, that is required for caspase activation. ${ }^{15,16}$ In our experiments we compared the kinetics of plasma membrane depolarization induced by oxidized cytochrome $c$ and dithionite-reduced cytochrome $c$ added to the cytosol by microinjection. In the time dependence of the change of $\Delta \Psi$, shown in Figure $3 \mathrm{~A}$, we observe a time lag of $\sim 20 \mathrm{~min}$ for the oxidized state of cytochrome $c$ relative to the reduced one. Small variations in the amount of cytochrome $c$ injected are unlikely to cause the lag as the kinetics of depolarization are insensitive to cytochrome $c$ concentration (inset, Figure $3 \mathrm{~A}$ ). A likely explanation of this observation is the requirement of intracellular reduction of cytochrome $c$ to ferrocytochrome $c$, which alone may activate caspase. To test this possibility we examined the time requirement for cytosolic reduction of oxidized cytochrome $c$. Optical absorption spectra of mitochondria-free cytosol extracts from oocytes injected with ferricytochrome $c$ (or oxidized cytochrome $c$ ) indicate that the heme chromophore initially stays oxidized. Figure 3B shows the spectrum of cytochrome $c$ of the cell-free extract prepared within $\sim 2$ min of microinjection. The Soret maximum of $409 \mathrm{~nm}$ indicates that cytochrome $c$ stays oxidized initially. Slow reduction, however, sets in and results in essentially complete reduction at longer times. For example, the spectrum recorded $60 \mathrm{~min}$ after microinjection, shown in Figure 3B, exhibits a red shift of the Soret maximum from 409 to $413 \mathrm{~nm}$ with a concomitant increase in the extinction coefficient together with the appearance of $Q$ bands at 520 and $549 \mathrm{~nm}$. This demonstrates the reduction of ferricytochrome $c$ to ferrocytochrome $c$. The time course of the reduction reaction was followed in cell-free extracts using the increase in absorbance at $549 \mathrm{~nm}$ (inset, Figure 3B). The kinetics yield a rate constant of $0.08 \mathrm{~min}^{-1}$. Similar observations of the reduction of cytochrome $c$ were made in cell free extracts prepared at different time intervals after injection of cytochrome $c$ into a batch of oocytes (data not shown). The time lag observed prior to depolarization of oocytes injected with ferricytochrome $c$ (Figure $3 \mathrm{~A}$ ) correlates well with the kinetics of reduction deduced from these experiments, and appears to suggest that ferricytochrome $c$ first undergoes reduction to ferrocytochrome $c$ in order to bring about the observed changes in plasma membrane potential. An immediate requirement then is to demonstrate directly the null effect of oxidized cytochrome $c$ in the induction of membrane depolarization and death. We have been unable to do this due mainly to the difficulty of maintaining the injected cytochrome $c$ in the oxidized state in vivo. Hampton et al. ${ }^{14}$ in their in vitro experiments used minimal quantities of cyanide to maintain the protein in its 
oxidized state to demonstrate its efficacy in activating caspases. We are currently grappling with the technical difficulties of using a similar approach in vivo.

\section{Failure of yeast iso-1-cytochrome $c$ in the induction of apoptosis}

We recorded the change in $\Delta \Psi$ of Xenopus oocytes following microinjection of yeast iso-1-cytochrome $c$. Figure $3 \mathrm{~A}$ shows no depolarization of membrane potential following microinjection of yeast iso-1-cytochrome $c$. This is consistent with earlier reports of the inability of this isoform of cytochrome $c$ to induce apoptosis (see, for example, reference ${ }^{9}$ ), and establishes the utility of the depolarization assay as an early indicator of caspase-activation. The ineffectiveness of yeast iso-1-cytochrome $c$ in initiating apoptosis still remains a puzzling issue because the yeast protein bears high structural similarity with eukaryotic cytochromes $c .^{17}$

\section{Induction of DEVDase activity by injected cytochrome $c$}

To ascertain that the death of Xenopus oocytes is indeed due to a canonical apoptotic pathway it is necessary to show that the injected cytochrome $c$ turns on the functional activity of aspartate specific proteases. ${ }^{2}$ Figure $3 \mathrm{~A}$ establishes that inhibition of caspase activity by Ac-DEVD-CHO prevents both the morphological and membrane potential changes caused by cytochrome $c$. More directly, we have followed the hydrolysis of the specific fluorogenic substrate Z-DEVD-AFC in cell-free extracts prepared from oocytes injected with cytochrome $c$. The time-dependence of the increase in the fluorescence of free AFC in the apoptotic cell-free extract, shown in Figure 4, suggests cytochrome $c$-induced activation of aspartate protease(s), and hence death due to apoptosis. The steady-state fluorescence emission spectrum of a mitochondria-free extract (inset, Figure 4), prepared from cytochrome $c$-injected cells, recorded after the addition of ZDEVD-AFC also indicates hydrolysis of the substrate and release of the fluorophore. The cell-free extracts prepared from oocytes injected with yeast iso-1-cytochrome $c$, however, produced no DEVDase activity, consistent with its inability to induce apoptosis. Also, extracts of cells which were injected with both cytochrome $c$ and Ac-DEVD-CHO failed to show Z-DEVD-AFC hydrolysis. These results show that the loss of membrane potential in cells injected with cytochrome $c$ is correlated with the initiation of the apoptotic cascade.

\section{Comparison of morphological changes and membrane depolarization}

Figure 2 presents the kinetics of the appearance of morphological changes on the surface of cells injected with cytochrome $c$. These provide a measure of the death of a population of cells and demonstrate the variability of response from cell to cell with some cells dying early and others surviving through the entire period of observation. On the other hand, the time dependence of the change of the resting membrane potential is similar for all microinjected cells, suggesting that the change in $\Delta \Psi$ is an early and general

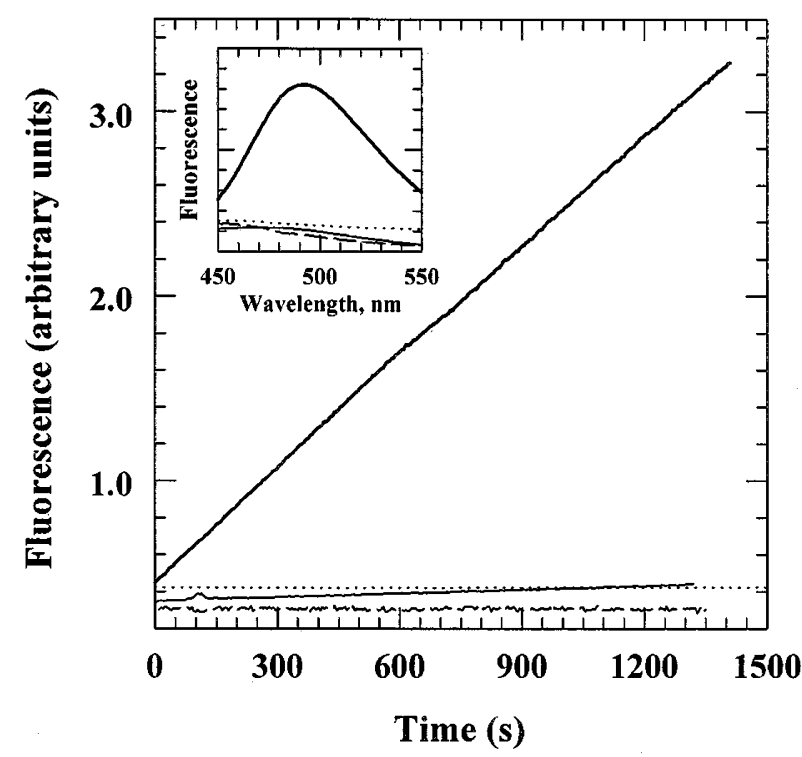

Figure 4 Assay of DEVDase activity in Xenopus oocyte extracts. Z-DEVDAFC was added to a final concentration of $\sim 150 \mathrm{nM}$. The release of AFC following hydrolysis of the substrate is monitored at $505 \mathrm{~nm}$, on excitation at $400 \mathrm{~nm}$. The extracts were prepared from 10-20 oocytes $\sim 3 \mathrm{~h}$ after injection of cytochrome c. $(-)$ cytochrome $c ;(\sim \sim \sim)$ yeast iso-1-cytochrome $c$; (- - - - ) cytochrome $c+$ Ac-DEVD-CHO $(90 \mathrm{nM})$; (-) cytochrome $c$ injected but no ZDEVD-AFC added in assay. The final intracellular concentration of injected proteins in all cases was $3 \pm 1 \mu \mathrm{M}$. Inset, a fluorescence spectrum, recorded $\sim 10 \mathrm{~min}$ after addition of Z-DEVD-AFC to the extract prepared from cytochrome $c$-injected oocytes

event during cell death. A decline in $\Delta \Psi$, however, may not mean that all metabolic activities including the functional activities of macromolecules would halt simultaneously, implying that the rate measured for the death of a single cell is probe dependent. Thus, the DEVDase activity can be monitored for a much longer period of time even though the membrane is depolarized sharply within $\sim 90$ min of injection of cytochrome $c$. In the context, it should be made clear that capases, at least upstream ones, are activated soon after the appearance of cytochrome $c$ in the cytosol, but their activity can be assayed at a much later time point partly due to their persistence in dying cells, and possibly due to delayed recruitment of downstream capases. Time dependent measurements of different physical and chemical probes following microinjection would help in establishing the chronology of events during cell death.

\section{Sequence of events during apoptosis}

How soon are caspases activated in response to cytosolic presence of cytochrome $c$ ? In cytochrome $c$-dependent death pathways translocation of cytochrome $c$ from mitochondria is essential for recruiting caspases into action. ${ }^{2}$ The direct delivery of exogenous cytochrome $c$ into the cytosol abolishes the need for a death signal and subsequent release of cytochrome $c$ from mitochondria. An important point to consider is the time interval between the appearance of cytochrome $c$ in the cytosol and the onset of 
caspase activity. We performed a set of experiments in which oocytes microinjected with cytochrome $c$ were flash frozen at different time intervals. Later, cell extracts of the frozen cells were prepared rapidly, and DEVDase activity was determined using the fluorescence assay. Figure 5 shows the results for eight time points $(0,7,12,17,22,27,32$, and $42 \mathrm{~min})$ after the injection. Clearly, caspases are activated within $7 \mathrm{~min}$ of cytosolic presence of cytochrome $c$, and Ac-DEVD-CHO inhibits the proteolytic activity. The family of time traces also shows that the rate of hydrolysis of Z-DEVD-AFC increases as the time interval between microinjection and freeze quenching increases (Figure 5). This is due most likely to multi-step recruitment of different caspases as apoptosis progresses. DEVD-AFC is cleaved primarily by caspase-3, but other active caspase such as caspase-6, -7, and -8 may also act on it. Since the downstream caspases are expected to be activated only at later times, the higher rate of hydrolysis of DEVD-AFC at times long after addition of cytochrome $c$ is not unexpected.

When does cell membrane depolarization occur in relation to other apoptotic events? Figure $3 \mathrm{~A}$ shows an interval of at least $20 \mathrm{~min}$ between microinjection and the onset of depolarization of the cell membrane, but we have seen that caspases are activated within $7 \mathrm{~min}$ of delivery of cytochrome $c$ into the cytosol (Figure 5). It, thus, becomes clear that membrane depolarization occurs well after caspase activation. Morphological changes associated with apoptosis are much later events. We have no data at the molecular level to trace out the exact sequence of events during apoptosis

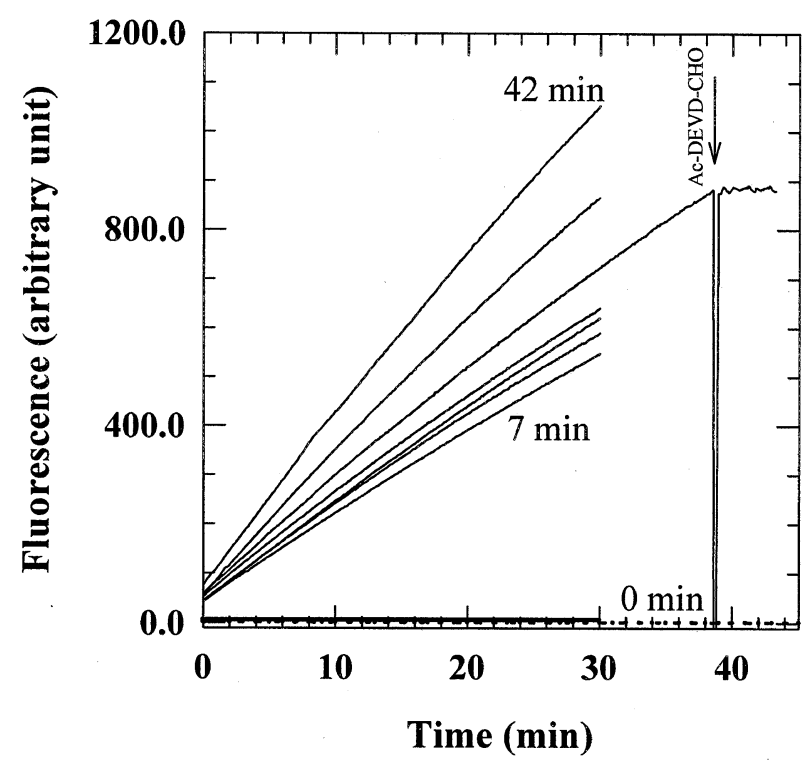

Figure 5 Assay of DEVDase activity in Xenopus oocyte extracts prepared at different time intervals following delivery of cytochrome $c(2 \mu \mathrm{M})$ into oocytes. Hydrolytic release of AFC monitored from oocytes frozen at $0,7,12,17,22$, 27,32 , and 42 min after injection of cytochrome $c$. Concentration of Z-DEVDAFC in the assay mixture was $150 \mathrm{~nm}$. To show that the AFC hydrolysis reaction is competitively inhibited by Ac-DEVD-CHO, the inhibitor was added to a final concentration of $80 \mathrm{nM}$ at the 35 th minute of the run of the extract made from oocytes frozen $27 \mathrm{~min}$ after injection of cytochrome $c$ leading to membrane depolarization. While we continue to work in this direction, the results presented in this study lead to a better understanding of the events and their temporal resolution in the cytochrome $c$-dependent death pathway.

\section{Summary}

The findings of this study are recapitulated in view of the canonical cytochrome $c$-dependent death pathway (see, for example, reference ${ }^{2}$ ) summarized below. Caspase activity appears within $7 \mathrm{~min}$ of the presence of cytochrome $c$ in the cytosol. Improved time resolution of our experiments may decrease this time interval. Plasma membrane depolarization, a later event, appears after about $30 \mathrm{~min}$, and in about $3 \mathrm{~h}$ the membrane loses its physical integrity. Finally, we stress that the plasma membrane depolarization is a discrete event associated with cell death. The single oocyte microinjection experiments and the electrochemical voltage probe can be used to monitor cell death. The effectiveness of any inhibitor or effector can be readily tested by injection into these oocytes simultaneously with cytochrome $c$ or at selected intervals thereafter.

\section{Materials and Methods}

\section{Materials}

Oocytes were extruded from the peritoneal cavity of anaesthetized Xenopus laevis through a ventral incision, and collected in ORMg medium (concentrations in $\mathrm{mM}$ : $\mathrm{NaCl} 82.5, \mathrm{KCl} 2, \mathrm{MgCl}_{2} 20$, HEPES 5, $\mathrm{pH} 7.5$ ). The cells were used within $24 \mathrm{~h}$ of collection, and with collagenase treatment (1 $\mathrm{mg}$ Sigma collagenase per $\mathrm{ml}$ of $\mathrm{ORMg}$ ). Horse heart cytochrome $c$ and yeast iso-1-cytochrome $c$ were obtained from Sigma (St. Louis), and an aqueous solution of the protein $(\mathrm{pH} \sim 7.5)$ was used without further purification. Reduced cytochrome $c$ was prepared by adding a minimal quantity of Sodium Dithionite to the aqueous solution of the protein. Excess dithionite was removed by passing the solution through a Sephadex G25 column equilibrated in water. The concentration of cytochrome $c$ was determined spectrophotometrically by using the extinction coefficient, $\varepsilon_{409}$, of $1.06 \times 10^{5} \mathrm{M}^{-1} \mathrm{~cm}^{-1} .{ }^{18}$ Other chemicals and reagents used were of highest purity.

\section{Microinjection of cytochrome $c$}

Typically, $46 \mathrm{nl}$ aqueous solutions of cytochrome $c$, contained in a capillary needle, was microinjected into each oocyte using a microinjector (Nanoject ${ }^{\mathrm{TM}}$, Drummond). For calculation of the final intracellular concentration of injected cytochrome $c$, the cytosolic volume of an oocyte was taken to be $\sim 1 \mu$ l.

\section{Measurement of membrane potential}

Resting membrane potential of cytochrome $c$-injected oocytes bathed in ND96 medium (concentrations in $\mathrm{mM}: \mathrm{NaCl} 96, \mathrm{KCl} 2, \mathrm{CaCl}_{2} 1.8$, $\mathrm{MgCl}_{2}$ 1, HEPES 5, pH 7.5) was recorded with an intracellular microelectrode attached to the voltage probe of a two-electrode voltage clamp amplifier (Warner Instrument Co., NJ, USA). The electrode was filled with $3 \mathrm{M} \mathrm{KCl}$ and typically had an initial tip resistance of $1 \mathrm{M} \Omega$. Transmembrane potentials were recorded at room 
temperature in a time-resolved manner. The dead-time of measurement defined as the time lapsed between the injection and the beginning of measurement was 1 to 2 min.

\section{Measurement of kinetics of reduction of ferricytochrome $c$}

The absorption spectra of cytochrome $c$ in cell free extracts (prepared in $100 \mathrm{mM}$ phosphate buffer, $\mathrm{pH}$ 7) was recorded in a Cary100 UVvisible spectrophotometer (Varian). The reduction kinetics were monitored at $549 \mathrm{~nm}$. To monitor kinetics of intracellular reduction of cytochrome $c$, cell-free extracts were prepared at specified times following injection and optical spectra recorded immediately.

\section{Assay of proteolytic activity of DEVDase}

About $3 \mathrm{~h}$ after the injection of cytochrome $c, 12$ oocytes were homogenized in the lysis buffer (50 mM HEPES buffer, $\mathrm{pH} 7.5$, containing $10 \%$ sucrose and $0.1 \%$ Triton $\mathrm{X}-100$ ). The use of PBS, $\mathrm{pH} 7.4$, instead of the lysis buffer did not affect the result. Following removal of cell debris and mitochondria from the homogenate by centrifugation, the supernatant was diluted 10 -fold with PBS, and incubated at $37^{\circ} \mathrm{C}$ for about $30 \mathrm{~min}$ in the presence of $1 \mathrm{mM} \mathrm{DTT} .4 \mu \mathrm{l}$ of a $50 \mu \mathrm{M}$ solution of Z-DEVD-AFC (Pharmingen), dissolved in DMSO, ${ }^{19}$ was added to the incubate in a quartz $1 \mathrm{~cm}$ square cell to obtain a final concentration of $125 \mathrm{nM}$. Fluorescence was measured in a SPEX Fluorolog 2 fluorometer (excitation: $400 \mathrm{~nm}$, and emission: $505 \mathrm{~nm})$.

\section{Acknowledgments}

We thank Dr. Apurva Sarin for many suggestions and for critical reading of the manuscript, and Dr. Aditya Kapil for help with experiments. This work was supported by internal funds from the NCBS.

\section{References}

1. Green DR (1998) Apoptotic pathways: the roads to ruin. Cell. 94: 695-698

2. Reed JC (1997) Cytochrome c: can't live with it - can't live without it. Cell. 91: $559-562$.

3. Liu X, Kim CN, Yang J, Jemmerson R and Wang X (1996) Induction of apoptotic program in cell-free extracts: requirements for dATP and cytochrome c. Cell. 86: $147-157$
4. Kluck RM, Bossy-Wetzel E, Green DR and Newmeyer DD (1997) The release of cytochrome $c$ from mitochondria: a primary site for $\mathrm{Bcl}-2$ regulation of apoptosis. Science. 275: $1132-1136$

5. Kuwana T, Smith JJ, Muzio M, Dixit V, Newmeyer DD and Kornbluth S (1998) Apoptosis induction by caspase- 8 is amplified through the mitochondrial release of cytochrome c. J. Biol. Chem. 273: 16589-16594

6. LiF, Srinivasan A, Wang Y, Armstrong RC, Tomaselli KJ and Fritz LC (1997) Cellspecific induction of apoptosis by microinjection of cytochrome c. J. Biol. Chem. 262: 30299-30305

7. Zhivotovsky B, Orrenius S, Brustugun OT and Døskeland SO (1998) Injected cytochrome $c$ induces apoptosis. Nature 391: 449-450

8. Neame SJ, Rubin LL and Philpott KL (1998) Blocking cytochrome $c$ activity within intact neurons inhibits apoptosis. J. Cell. Biol. 142: 1583-1593

9. Garland JM and Rudin C (1998) Cytochrome c induces caspase-dependent apoptosis in intact hematopoietic cells and overrides apoptosis suppression mediated by bcl-2 growth factor signaling, MAP-kinase and malignant change. Blood. 92: 1235-1246

10. Yang J, Liu X, Bhalla K, Kim CN, Ibrado AM, Cai J, Peng TI, Jones DP and Wang X (1997) Prevention of apoptosis by Bcl-2: release of cytochrome c from mitochondria blocked. Science. 275: 1129-1132

11. Takano T and Dickerson RE (1981a) Conformation changes of cytochrome c. I. Ferrocytochrome cstructure refined at 1.5 Å resolution. J. Mol. Biol. 153: 79-94

12. Takano T and Dickerson RE (1981b) Conformation changes of cytochrome $c$. Il. Ferricytochrome $c$ refinement at $1.8 \AA$ and comparison with the ferrocytochrome structure. J. Mol. Biol. 153: 95-115

13. Berghuis AM and Brayer GD (1992) Oxidation state-dependent conformational changes in cytochrome c. J. Mol. Biol. 223: 959-976

14. Hampton MB, Zhivotovsky B, Slater AFG, Burges DH and Orrenius S (1998) Importance of the redox state of cytochrome $c$ during caspase activation in cytosolic extracts. Biochemistry 328: 95-99

15. Pan Z, Voehringer DW and Meyn RE (1999) Analysis of redox regulation of cytochrome $c$ induced apoptosis in a cell free system. Cell Death Differ. 6:683688

16. Ma Y, Ogino T, Kawabata T, Li J, Eguchi K and Okada S (1999) Cupric nitrilotriacetate-induced apoptosis in HL-60 cells association with lipid peroxidation, release of cytochrome $c$ from mitochondria and activation of caspase-3. Free Radic. Biol. Med. 27: 227-233

17. Louie GV and Brayer GD (1990) High resolution refinement of yeast iso-1cytochrome $c$ and comparisons with other eukaryotic cytochromes $c$. J. Mol. Biol. 214: $527-555$

18. Babul J and Stellwagen E (1972) Participation of the protein ligands in the folding of cytochrome c. Biochemistry 11: 1195-1200

19. Sarin A, Wu ML and Henkart PA (1996) Different interleukin-1 $\beta$ converting enzyme (ICE) family protease requirements for the apoptotic death of $T$ lymphocytes triggered by diverse stimuli. J. Exp. Med. 184: 2445-2450 